This item was submitted to Loughborough's Research Repository by the author.

Items in Figshare are protected by copyright, with all rights reserved, unless otherwise indicated.

Changes in specific absorption rate in the head due to metallic gap loops and a simulated mobile phone source in a study of the effects of jewellery

PLEASE CITE THE PUBLISHED VERSION

PUBLISHER

(C) Institute of Electrical and Electronics Engineers (IEEE)

LICENCE

CC BY-NC-ND 4.0

REPOSITORY RECORD

Whittow, W.G., C.J. Panagamuwa, R.M. Edwards, Shahid Bashir, and J.C. Vardaxoglou. 2019. "Changes in Specific Absorption Rate in the Head Due to Metallic Gap Loops and a Simulated Mobile Phone Source in a Study of the Effects of Jewellery". figshare. https://hdl.handle.net/2134/3341. 
This item was submitted to Loughborough's Institutional Repository by the author and is made available under the following Creative Commons Licence conditions.

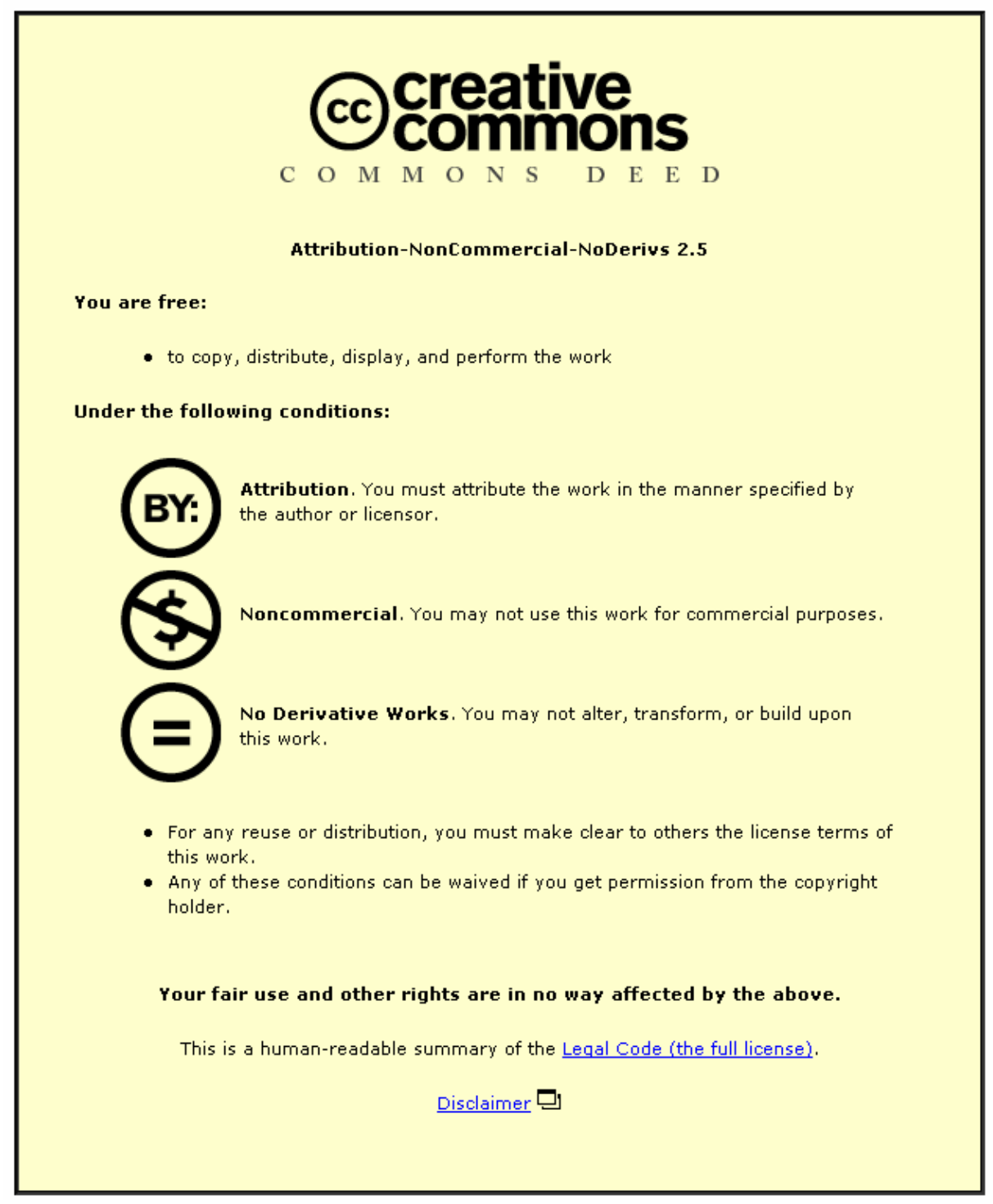

For the full text of this licence, please go to: http://creativecommons.org/licenses/by-nc-nd/2.5/ 


\title{
CHANGES IN SPECIFIC ABSORPTION RATE IN THE HEAD DUE TO METALLIC GAP LOOPS AND A SIMULATED MOBILE PHONE SOURCE IN A STUDY OF THE EFFECTS OF JEWELLERY
}

\author{
W. G. Whittow (1), C. J. Panagamuwa (1), R. M. Edwards (1), S. Bashir (1) \\ and J. C. Vardaxoglou (1) \\ (1) Department of Electronic \& Electrical Engineering, Loughborough University, \\ Leicestershire, LE11 3TU. UK. \\ Email:W.G.Whittow@lboro.ac.uk,Email: C.J.Panagamuwa@lboro.ac.uk \\ Email:R.M.Edwards@lboro.ac.uk,Email: S.Bashir@lboro.ac.uk
}

\begin{abstract}
This paper investigates Specific Absorption Rates (SAR) in the human head due to partial metallic gap loops (incomplete circular jewellery rings) at $1800 \mathrm{MHz}$. A Finite-Difference Time-Domain (FDTD) code was used to analyse different sizes and positions of circular rings with a section removed, near a homogenous cubic phantom with a dipole excitation. These partial loops significantly increased the SAR. Measurements from a DASY4 measurement system as well as commercial code results are used for validation. The FDTD code has also been used to analyse the effects of metallic jewellery gap loops when added to the nose of an anatomically realistic digital human head with a realistic source. A monopole on a box was placed in front of the face to represent a PDA. The gap loops on the nose of the realistic head behaved similarly to those near the cubic head. Gap loops of different sizes resonated when their orientation were changed. This means that smaller sizes of jewellery, in certain geometries, may resonate when used with mobile communications equipment than was previously expected.
\end{abstract}

\section{Introduction}

Recent estimates suggest there are over a billion mobile phones users in the world. Trends in technology mean that current and future mobile communications devices may be more commonly used in front of the face in a personal data assistant configuration. There has been both public and scientific comment that the radiation from mobile devices may adversely affect human health and a first stage to studying any possible problems is to establish the likely levels of radio frequency radiation in the head. Many of the people who use communications enabled devices may also wear metallic jewellery or have metallic objects in close proximity to their head. In previous works, the authors [1,2] have found that metallic spectacles can increase the SAR in the eyes and head. Please see $[1,2]$ for a more complete literature review of bioelectromagnetics and the effects of metallic objects on SAR. Recently, the authors [3] showed that metallic rings, also known as magnetic dipoles, resonate and increase the SAR when the circumference is approximately one wavelength. This equates to a diameter of $52 \mathrm{~mm}$ at $1800 \mathrm{MHz}$. This is applicable to spectacles and ear rings, but is less likely to be relevant to other types of facial jewellery. Please see [4] for an example of a gap loop antenna. This paper quantifies the effects on SAR due to gap loops; see Figure 1.

\section{Description of model}

An independent 3D FDTD code $[1,2]$ has been written. Perfectly Matched Layers (PML) absorbing boundary conditions are used to terminate the grid. The PML is eight cells thick and is positioned at least twelve cells from the head. The Yee cell size used throughout this paper is $2 \mathrm{~mm}$. The lowest number of cells per wavelength was always greater than ten. To allow comparison between simulations and measurements, a Z directed dipole model has been used. The dipole is fed at its centre with a sinusoidal CW source. The tangential E-field components are set to zero along the length of the dipole [1]. The dipole in the FDTD simulations is $74 \mathrm{~mm}$ long.

All results in this paper are normalised to $1 \mathrm{~W}$ input power and the frequency of excitation is $1800 \mathrm{MHz}$. The source was vertically orientated along the $\mathrm{Z}$ axis, $80 \mathrm{~mm}$ in front of the face in the $\mathrm{X}$ direction ad the $\mathrm{Y}$ axis was from ear to ear. The metallic gap loops were modelled using metallic Yee cells, by setting the conductivity of the cells equal to the conductivity of copper [1]. The gap loops investigated in this report were $2 \mathrm{~mm}$ thick (1 Yee cell) and of different diameters. The gap loops were created by creating a circular loop and then removing the outer edge on one side, see Figure 1 (b). SAR is the standard criteria to measure the amount of electromagnetic energy absorbed in the body and is calculated as in equation (1) where $|E|$ is the rms 
magnitude of the electric field strength vector, $\rho$ is the mass density of the material in $\mathrm{kg} / \mathrm{m}^{3}$ and $\sigma$ is the electrical conductivity in $\mathrm{S} / \mathrm{m}$. The SAR is calculated with the twelve-field approach [1].

$$
S A R=\frac{\sigma|E|^{2}}{\rho}
$$

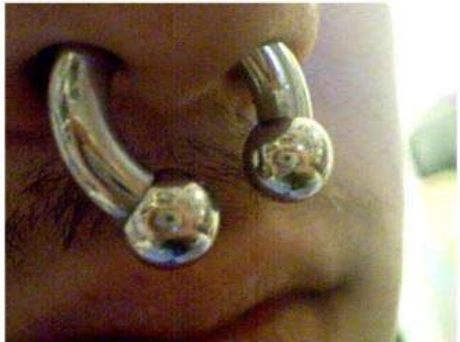

(a)

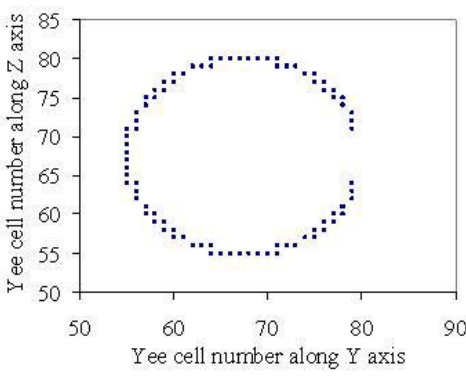

(b)

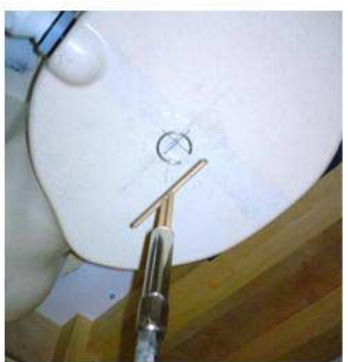

(c)

Figure 1. (a) An Example of a metallic jewellery gap loop, (b) a 52mm diameter $\subset$-shaped gap loop made from Yee cells and (c) the measurement setup.

\section{Results}

Initially, the FDTD code was used to search for the maximum effects in terms of SAR of adding a metallic gap loop near to a $200 \mathrm{~mm}$ cubic homogenous phantom, with a Z-directed dipole $80 \mathrm{~mm}$ away. The properties of the brain simulating tissue of the homogeneous head are $\left(\sigma=1.37 \mathrm{~S} / \mathrm{m}, \varepsilon r=40.48, \rho=1000 \mathrm{~kg} / \mathrm{m}^{3}\right)$. The brain fluid is surrounded by a $2 \mathrm{~mm}$ thick fibreglass shell $(\sigma=0 \mathrm{~S} / \mathrm{m}, \varepsilon r=3.5)$. Since the cubic head has a flat face our simulated jewellery can be made to lie fully in a parallel plane. Thus, our simulated results can be compared with DASY4 measurements made with the flat section of the SAM twin phantom [5] which can be thought of as a control. The length of the dipole used in the measurements was $72.5 \mathrm{~mm}$ and had a diameter of $3.66 \mathrm{~mm}$ [6].The centre of the dipole was placed below the centre of the loop, see Figure 1 (c) for the equivalent measurement setup.

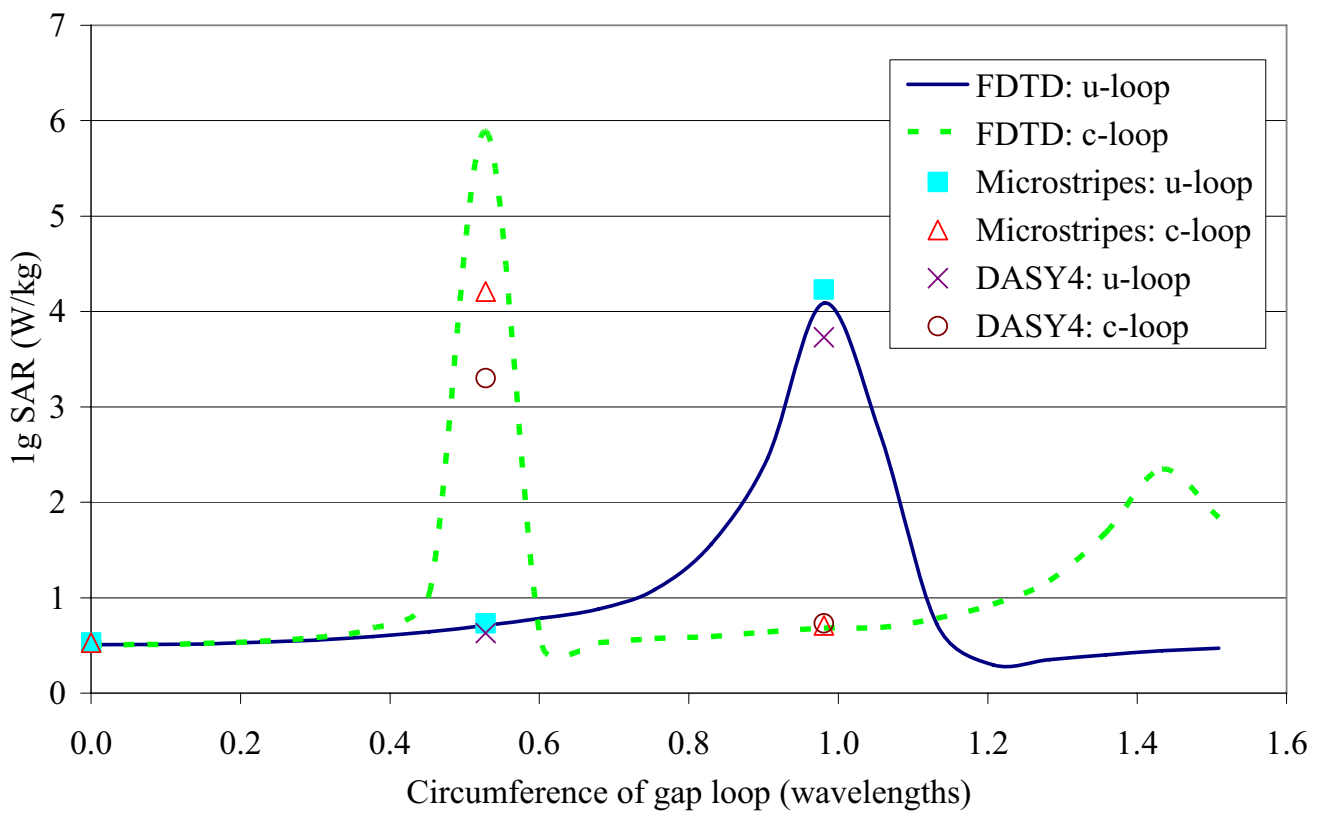

Figure 2. The 1g SAR in a homogeneous cubic phantom with different sizes and orientations of gap loops.

The results are shown in Figure 2 for different sizes and orientations of gap loops. Note, when defining our circumference (in wavelengths) we do not subtract the gap. It was found that when the gap in the loop was aligned near the end of the dipole source, the loop resonated at approximately one wavelength. We will refer to this as a $\cup$-loop. [3]. However, when the gap was positioned to the side of the dipole then the loop was seen to 
resonate at approximately half a wavelength. We will refer to this as a $\subset$-loop. The $\subset$-loop increased the SAR when the length of the loop without the missing section was approximately $0.44 \lambda$. The $\subset$-loop with a diameter of $28 \mathrm{~mm}$ increased the $1 \mathrm{~g}$ SAR by over eleven times. The $10 \mathrm{~g}$ SAR in the cube behaved in a similar way except the magnitudes were half the $1 \mathrm{~g}$ SAR values. The resonant $\subset$-loop increased the $10 \mathrm{~g}$ SAR by nine times. The FDTD results agreed well with Microstripes commercial software [7] and DASY4 measurements [5]. The agreement is excellent with the $\cup$-loop and good with the $\subset$-loop. The $1 \mathrm{~g}$ SAR results were found to be sensitive to how much of the loop is removed with the $\subset$-loop. We feel this may be because of more dominant end effects.

Figure 2 shows how different orientations of the gap loop behave differently. Further analyse of this was done with Microstripes [7]. The $\cup$-loop behaved like the complete loop shown in Figure 3 (a) and (b). With a vertical dipole, the maximum currents on the $\cup$-loop are on the sides of the loop. At resonance currents supported on the metal induce large magnetic fields at the sides of the loop and large electric fields at the top and bottom of the loop. Therefore, if a section of the loop is removed where the currents on the ring are at a minimum, then removing that section has little effect. Figure 3 (c) and (d) show the effect of removing the side of the loop where the currents are at a maximum to form a $\subset$-shaped gap loop. This changes the behaviour of the loop so that it behaves more like a pin than a loop [6]. The maximum current occurs opposite the gap, see the magnetic fields in Figure 3 (c) and the maximum electric fields occur at the gap in the loop.

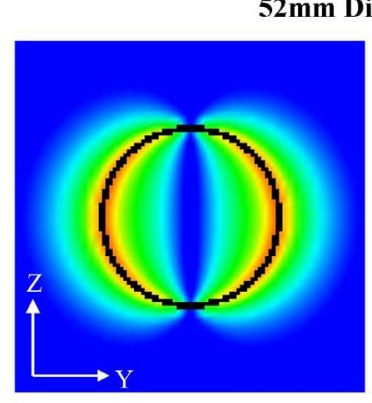

(a) RMS Magnetic Field $(\mathrm{dB})$

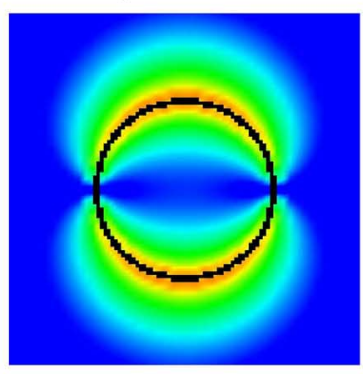

(b) RMS Electric Field (dB)

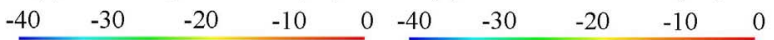

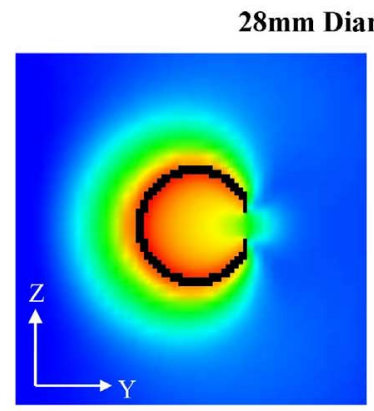

(c) RMS Magnetic Field (dB)

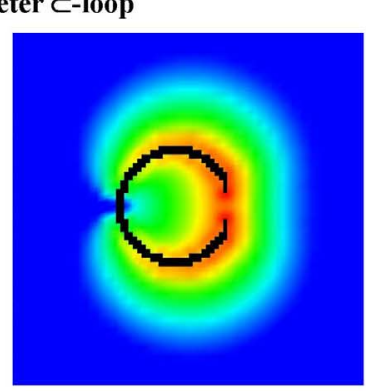

(d) RMS Electric Field (dB)

Figure 3. The magnetic and electric fields with a $52 \mathrm{~mm}$ loop and a $28 \mathrm{~mm} \subset$-shaped gap loop.

Metallic gap loops were also added to an anatomically realistic digital human head. An adult male head matrix provided by Brooks Air Force was used (http://www.brooks.af.mil/) [1]. The head is based on The Visible Human Project. It has 25 tissue types and has a $2 \mathrm{~mm}$ resolution. The densities and dielectric properties of the tissues in the Brooks head at $1800 \mathrm{MHz}$ are shown in [2]. The excitation in this section was a monopole positioned at the centre of the top face of a PEC box, positioned $80 \mathrm{~mm}$ in front of the tip of the nose. The box was $80 \mathrm{~mm}$ high, $40 \mathrm{~mm}$ wide and $20 \mathrm{~mm}$ deep. The gap loops touched the underneath of the tip of the nose.

The $1 \mathrm{~g}$ SAR results with different sizes of loop are shown in Figure 4. The Q factor was found to be less sharp than with a cubic head. This emphasizes the importance of initially using a simple cubic head to understand the mechanisms and validate the effects with measurements, however, the analysis must then be extended to a more realistic head model. When the gap loop touched the nose, the loop had different effects depending on the size and orientation of the loop. The $\subset$-loop increased the SAR by 5 times when the circumference (including the gap) was $0.45 \lambda$. An $\cap$-loop resonated at $0.75 \lambda$ while the $\cup$-loop resonated when the circumference was approximately a wavelength. Note, that the resonance sizes are smaller than with loops near the cubic head. We believe this is because the loop touched the nose and therefore the nose dielectrically loaded the loop. The $\cup$-loop has less contact with the nose and therefore, the dielectric loading effect is reduced. Note, similar effects have been found with gap loops below a cubic nose attached to a cubic head [8].

The effect of piercing the gap loop through the nose was investigated by moving the gap loops $4 \mathrm{~mm}$ in the $\mathrm{X}$ axis ( 2 cells into the head). This meant that part of the metallic loop replaced tissues in the skin. Moving the loop towards the head and piercing it though the skin generally reduced the maximum $1 \mathrm{~g}$ SAR, but the pierced loop still increased the $1 \mathrm{~g}$ SAR by 4 times, compared to without the loop. Note, that the $1 \mathrm{~g}$ SAR without the loop occurs in the tip of the nose whereas the $10 \mathrm{~g}$ SAR occurs at the back of the nose; therefore, the gap loops placed at the tip of the nose had less effect on the maximum $10 \mathrm{~g}$ SAR in the head. 


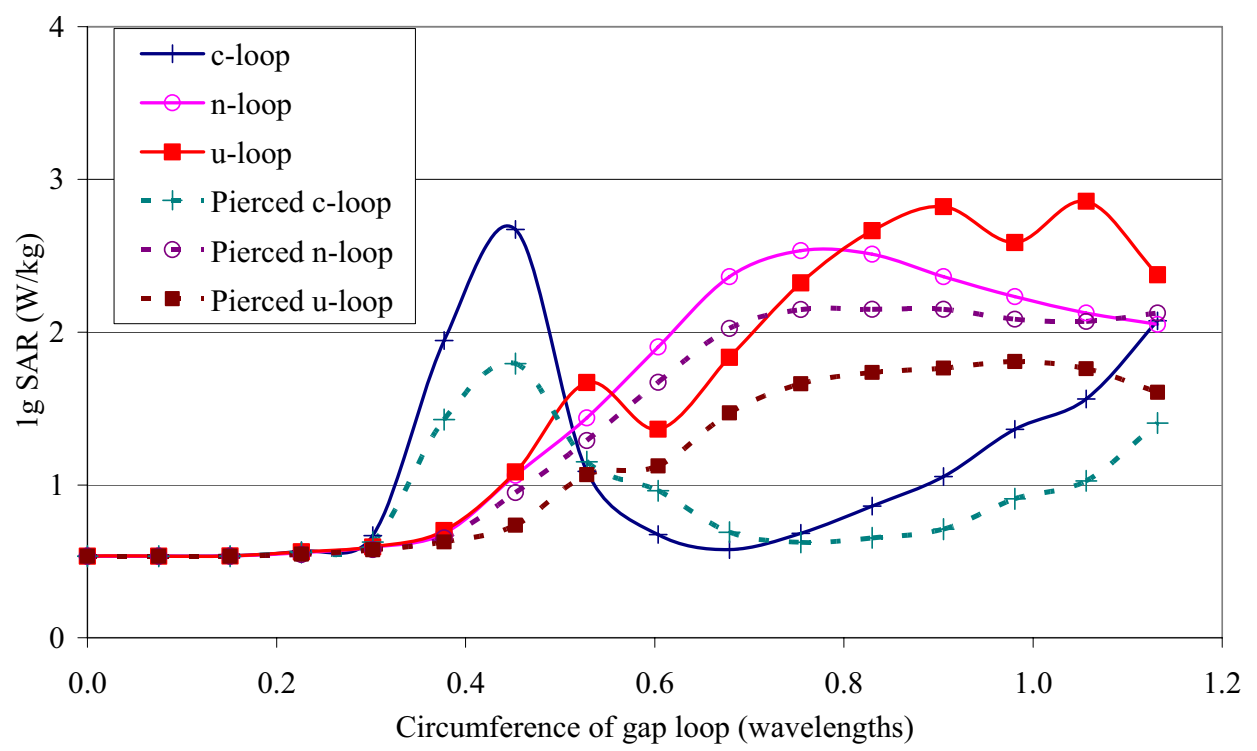

Figure 4. The 1g SAR in the Brooks head with different sizes and orientations of jewellery gap loops.

\section{Conclusions}

The FDTD method was used to investigate the SAR in the presence of circular jewellery gap loops. The $\cup$-loop behaved like a magnetic dipole and caused the largest SAR in the head when the loop was approximately one wavelength in circumference, whereas the $\subset$-loop, behaved more like a pin, resonating when the circumference was $\sim 0.5 \lambda$. This shows that the orientation of the loop is significant and that real sources with cross polar components would produce a combination of the effects mentioned here. Simulations agreed well with commercial software and DASY4 measurements.

Gap loops were also added to an anatomically realistic head with a realistic source. The gap loops increased the $1 \mathrm{~g}$ SAR by up to 5 times. Different orientations of gap loops resonated at different sizes. Moving the loop towards the head so that the loop was pierced through the skin reduced the effect. The results in this paper are also relevant to other facial jewellery as well as ear rings with a mobile phone positioned by the ear.

\section{References}

[1] W. G. Whittow and R. M. Edwards, "A study of changes to specific absorption rates in the human eye close to perfectly conducting spectacles within the radio frequency range 1.5 to $3.0 \mathrm{GHz}$," IEEE Trans. Antennas and Propagation, vol. 52, pp. 3207-3212, 2004.

[2] W. G. Whittow and R. M. Edwards, "Applications of a genetic algorithm for identification of maxima in specific absorption rates in the human eye close to perfectly conducting spectacles," IEE Proceedings Science, Measurement \& Technology, vol. 152, pp. 89-96, 2005.

[3] W. Whittow, C. Panagamuwa, R. Edwards, and J. Vardaxoglou, "Specific absorption rates in the human head due to circular metallic earrings at $1800 \mathrm{MHz}, "$ in 2007 Loughborough Antennas and Propagation Conference, Loughborough, UK, 2007, pp. 277-280.

[4] R. L. Li, E. M. Tentzeris, J. Laskar, V. F. Fusco, and R. Cahill, "Broadband loop antenna for DCS1800/IMT-2000 mobile phone handsets," IEEE Microwave and Wireless Components Letters, vol. 12, pp. 305-307, 2002.

[5] T. Schmid, O. Egger, and N. Kuster, "Automated E-field scanning system for dosimetric assessments," IEEE Microwave Theory and Techniques, vol. 44, pp. $105-113,1996$.

[6] W. Whittow, C. J. Panagamuwa, R. Edwards, J. C. Vardaxoglou, and P. McEvoy, "A study of head worn jewelry, mobile phone RF energy and the effect of differing tissue types on rates of absorption," in The First European Conference on Antennas and Propagation (EuCAP 2006), Nice, France, 2006.

[7] Flomerics, "Micro-Stripes Reference Manual, Release 7.5," 2006.

[8] W. G. Whittow, C. J. Panagamuwa, R. M. Edwards, and J. C. Vardaxoglou, "Report 12: Effects of incomplete circular loops (arcs) on SAR in the human head," Loughborough University, UK. EPSRC Grant No. EP/C517490/1. EPSRC Grant Title: A Study Of RF SAR Rates In The Human Head Due To Cellular Enabled Personal Data Assistants, Spectacles and Jewellery, Sept 2007. 